\section{Oriatinal Communications.}

\section{GENERAL CONSIDERATIONS RESPECTING FEVER}

By C. Handrieid Jones, M.B., F.R.S., Physician to St. Mary's Hospital.

Is the following propositions and remarks, an attempt is made to take a general view of the principal phenomena of the febrile state, regarding their pathology from the point of view so well indicated by Parkes and Virchow. I must suppose the fundamental facts ascertained by Bernard to be well known, and will only refer to the article "Sympathetic Nerves", in the Cyclopadia of Anatomy and Physiology, for a resume of them and to the British and Foreign Medico-Chirurgical Review, $185 \pi$, for a notice of the analogous and confirmatory experi ments performed by Jaschkowitz on the splenic, and by Samuel on the coliac plexus. I am far from imagining that we have yet any complete and satisfactory theory of fever; but I cannot think that any one can have any just conception or enlarged view of the pathology of fever, who ignores the capital facts so well elaborated by Bernard.

1. Fever may result from pure nerrous exhaustion. I am acquainted with two medical men who have suffered attacks of fever, to all appearance, in consequence of fatigue in walking. In such cases, it may be presumed that the sympathetic system has become affected, as well as the cerebro-spinal.

2. The nervous power of the cerebro-spinal system may be extremely depressed without fever being induced.

3. Paralysis of the vaso-motor (sympathetic) nerves is probably essential to all fevers.

4. In the majority of fevers, the nerrous power of the cerebro-spinal system is greatly debilitated or impaired.

5. In all varieties of asthenic fever, debility and depression predominate. They may be more marked in some organs than in others, and may be variously combined with degrees of irritation; but still they give its character to the disorder.

6. All debilitating influences, in their less severe degrees, tend to produce a state of irritability ; in their more violent action, they cause prostration. The character of irritability is weakness, together with an undue sensitiveness to all stimuli. In such states, there is often a great display of force ("increased action"); but it is to be remembered that this takes place at the expense of the radical* forces of the economy, which are drawn upon for the excessive development of the acting. Wild delirium and increased energy of circulation can only occur at the expense of the real porrer of the brain and heart.

\%. In the sthenic and inflammatory fevers, the heart's action is excited, and increased in force, as well as in frequency. The cause of this may be presumed to be, that the blood, being hotter than natural, stimulates the heart unduly. The place of the heart in the vital economy, its highly developed structure, its peculiar power of not knowing fatigue, unlike other muscles, afford sufficient reason why it should be stimulated to in creased energy of action, while the arterial muscles are more or less relaxed or paralysed. It has a higher responsive faculty. At the same time, the tissues of the body generally are not so much debilitated as in asthenic fever.

8. The causation of pyrexia attending on local inflammations may be viewed as follows. The blood traversing the in flamed part becomes altered in some way (perhaps by having an increase of fibrine generated in itself), so that it comes to contain matter, which may be compared to the miasm of fevers (idiopathic), like it producing irritating and debilitating effects 'This view, which is Andral's, is adopted by Mr. Erichsen and Dr. Markham.

9. It may appear a contradiction to the above statements, that, in the more sthenic idiopathic fevers, and in certain pyrexiæ attending on inflammation, especially in the case of serous membranes, the radial artery is not paralysed, but

* For remarks on this important distinction, see Trousseau, Materia Medica, vol. $i$, p. 42 . rather contracted; the pulse is hard or wiry. This would not certainly prove that the swollen arteries are in the same state, but let it be granted that it does. Observation of the results of dividing the sympathetic nerve in the neck shows that relaxation of arteries and increase of temperature are not inseparably connected. The hyperæmia which ensues after the operation diminishes considerably in a day or two, though the elevation of temperature persists. Moreover, in repeating the experiment upon a cat, I found that the temperature became greatly elevated, without the existence of much apparent hyperæmia. It certainly did not appear that the increased heat could be accounted for by the hyperæmia. It is Bernard's opinion that the temperature is not augmented solely in consequence of the part lying in the range of the paralysed nerves receiving more blood, but that there is actually an altered state of the nutrition of the part. Brown-Séquard and Walter, on the other hand, ascribe the increased temperature solely to the increased afflux of blood. The circumstance that the temperature of the side operated on is sometimes $2^{\circ}$ or $3^{\circ} \mathrm{Fahr}$. higher than that of the internal parts is materially in favour of Bernard's view. So are also the phenomena of phlegmasia dolens, and some analogous white inflammations (as Dr. Graves calls them), in which, at the same time that there is swelling and increased heat, the pallor certainly indicates that the arteries are constricted. In some cases, it may be that the vaso-motor nerves are so affected, so debilitated, that increased heat is produced by the derangement and hurry of the nutrition processes, while yet the arterial muscles retain power enough to be stimulated to contraction by the over-heated blood. Their contraction then might be explained in the same way as the increased action of the heart.

10. The accelerated action of the heart in fever (i.e., the in creased rapidity, not force, of its contractions) may very plau. sibly be explained by considering the medulla oblongata or pneumogastric nerve to be in a debilitated state. It is not in deed so fully established that section of the pneumogastric causes increased frequency of the heart's action, as it is that stimulation of it or of its origin retards or arrests it; but the conclusion is at least eminently probable. The rapid action of the heart, the palpitation, and breathlessness, which occur in anæmic girls, and males not anæmic but of weak tone, on any sudden muscular exertion, are more probably owing to debility of the par vagum and medulla oblongata than to any other cause. If a man in vigorous health attempt a feat of running or swimming, without having practised for a length of time, he will surely find that his " wind" speedily fails him; he will have much palpitation and panting; but if he is in training, he can bear the exercise without being blown. In this case, the inproved power can scarcely be attributed to anything except the increased energy of the nervous system. The hurried action of the heart, as is well known, is in no wise of the essence of fever; paresis of other centres than the regulating cardiac is necessary to produce this effect.

11. In the more sthenic forms of fever and inflammatory pyrexia, the diminution of the cutaneous and urinary secretions, and of the salivary, is a phenomenon sufficiently constant to require notice. It stands in sharply defined contrast to the profuse flow which is common in states of debility. In various conditions, of which low nervous power is a prominent feature, a copious flow of aqueous urine is a common occurrence; and its connexion with some depressing mental emotion is often very apparent, as in the hysterical paroxysm, or the case of hypochondriasis related by Sydenbam (Sydenbam's Society's edition, vol. ii, p. 93). Profuse sweating during sleep is a common occurrence in aguish disorder, withont any organic disease or regular fit. Salivation may occur as one of the manifestations of malarious disease. In the case of the kidneys, it is certain that there is not solely increased activity of the glandular tissue, but that the homogeneous membrane of the Malpighian tufts must be in some way altered, so as to allow the more free permeation of aqueous fluid. The same is probably the case with other glands. Now, in the sthenic febrile state, the reverse prevails; the hon:ogeneous membranes are much less permeable by water than asual. In the sthenic fevers, again, this retention of aqueous fluid is not observed; indeed, the limiting membranes allo $N$ the transudation not only of water, but also of albuminous : natter and fibrine dissolved in it, and even of blood. On what this difference in the filtering power of the limitary membranes depends, is quite a matter of guess; it seems, however, not imp robable that it is in part dependent on the amount of fibrine in the blood. In the sthenic inflammations, the amount of fibrine is notably increased; and in these also the diminution of the secretions is, as a rule, most ob. 
servable. The fibrinous casts of the renal tubes are often so purely homogeneous, that the idea seems naturally suggested that the limitary membranes may be strengthened and thickened by additions of this substance when it is circulating in excess. It is, however, certain that the filtering power of these membranes may be notably affected by variations in nervous influence. There appears to be a general accordance between the behaviour of the arterial coats and the capillary walls. In relaxed states of the contractile coat of the arteries, the capillary membranes are more permeable than usual, and vice versâ. The relaxation of the latter may proceed to that extreme degree in which they allow blood-corpuscles to pass through the softened texture, and ecchymosis or hæmorrhage occurs. have seen this twice in aguish disorder, as subconjunctival effusion.

12. The liability of the various organs and tissues to asthenic inflammation during the course of fever probably depends on their vital power having been so lowered by the action of the poison that a little hyperæmic afflux becomes a cause of irritation. The case is the same as when a part has been frostbitten, and the circulation has been restored too rapidly. On the same ground, when the sympathetic is cut in the neck of a debilitated animal, severe conjunctivitis sometimes ensues, because the enfeebled tissue cannot withstand the stimulus of the hyperamia, intensified by the loss of the influence of the vaso-motor nerves. The diarrhœa of intestinal typhoid, and its follicular deposits, seem to me to be well explained in this way.

13. An occurrence which I think is more frequent in malarious fever, but which Dr. Corrigan speaks of as not unfrequent in typhus, or rather as a sequela of typhus, of late years, is jaundice; the skin and urine being deeply tinged, though the flow of bile into the intestine goes on freely. In this case, it may be presumed that the hepatic plexus is paralysed, and the liver in the same state as the side of the head when the cervical sympathetic is divided. Bile is secreted in excess, and a secretion-flux takes place. 'This, however, would not occur if the vitality of the liver were much depressed; the result would be rather inflammation. Turpentine, which cures the jaundice, acts no doubt in the same way as when it arrests a gastric hæmorrhage, stimulating the relaxed vessels to contract through the medium of their nerves.

14. 'The treatment of fever is to be ruled essentially by dis. criminating observation of the predominant pathological state, whether this approach nearer to excitement and irritation, or to pure debility and asthenia. In the former case, it may be needful to bleed largely, as in tropical fevers (vide Dr. Copland, Fever, p. 930); to give tartar emetic and opium, as Graves did in typhus with violent delirium; or apply leeches to the temples, as Dr. Corrigan recommends in states of insomnia; or, as we so often do in the diarrhœa of typhoid, to leech the region of the cæcum, and give hydrargyrum cum cretâ and Dover's powder. All these are instances where lowering means are employed, with guarded caution, to quiet excited action. In the latter case, quinine, wine, and brandy, are to be administered freely, according to the requirement of the individual case. Dr. Stokes', Dr. Brinton's, and Dr. Shute's recorded experience is quite in accordance with my own. I may add, that I cannot but think the old idea that brandy and wine act beneficially by their stimulant effect on the nervous system, is far more satisfactory than the chemical notion that they act by affording a ready fuel to the respiratory process. It is the nervous system which most of all feels the effect of the poison; and it is reasonable to address our remedies chiefly to it.

15. In the treatment of pyrexia attending on local inflammation, it is impossible to prevent the effect while the cause continues. Any tonic or stimulant that could be administered to stay the paralysis of the vaso-motor nerves, would act injuriously as an irritant upon the inflamed tissue, impede excretion, and increase the existing mischief. Thus, in febrile eczema, arsenic aggravates the skin-disease and the associated pyrexia. As long as the inflammation is of sthenic character, we must apply our efforts to reduce it; in the case of eczema, we leech the skin, or apply lead lotion, and give saline aperients. When, however, the inflammation has become asthenic, or is so from the first, there is either no fever, or it will yield with the inflammation to tonics. Thus, non-febrile eczema is often cured by arsenic, which probably tones the cutaneous vaso-motor nerves, and so contracts the arteries of the inflamed part. The distinction between sthenic and asthenic inflammation, as to the effects of treatment, is all important, and seems sadly lost sight of in the blood-letting controversy.

\section{NOTES OF CASES OF GUN-SHOT. WOUND.}

By G. Nayler, Esq., Assistant Surgeon, Central India Field Force.

Among the large number of wounded occasioned by the operations of the field force at Jhansi, a considerable proportion consisted of gun-shot cases. The opportunity was afforded me of witnessing many of them soon after their admission, and of watching their progress from day to day. On being subsequently transferred to the Field Hospital, they came under my care. As these cases constitute a class of injuries not often met with in civil practice, I have ventured to give the notes of some, taken at the bed-side, as well as the post mortem appearances.

To Dr. Ward, Surgeon of the 3rd European Regiment, under whom the following patients were admitted, I am indebted for much information and assistance.

Case I. Gun-shot Wound of the Chest; Ball Lodged. P. Williams, aged 20, was brought into Hospital at 8 A.Mr. on April 3. A bullet had entered the chest close to the inner border of the scapula, and about an inch above its spinous process. No further trace of the ball could be detected on examination. The patient complained of some pain in the vicinity of the wound, but did not appear to suffer much distress. The pulse was 80 , soft ; there was slight dyspnœa. $\mathrm{He}$ was ordered to have an ounce of saline mixture every four hours, and spoon-diet. Lint and bandages were applied to the wound.

April 6 . The lint was removed, and simple water-dressing was substituted. Slight suppuration now commenced. No other medicines than salines were given, except an occasional dose of castor oil, as he was progressing favourably, until

April 10. He was now suffering from much pain, referred to the right side of the chest, and to the left scapula, where the pain appeared to be fixed, and the part tender. Pulse quick, skin hot. Pain was felt in taking a deep inspiration. He lay on the right side. $\mathrm{He}$ had much thirst. Low diet was ordered; three dozen leeches were applied to the region of the left scapula; and a saline effervescing draught was given every four hours.

4 P.s. There was dulness on percussion over the posterior aspect of the right lung in its lower and middle portions, and also over the left scapular region, extending in a line to this spot from the right side. Little air was heard to enter in these situations; but in the front of the chest the breathing did not seem to be affected. He still lay in the same position. The pulse was quick; the thirst was urgent. Two dozen leeches were applied between the scapulæ. A pill of three grains of calomel and half a grain of opium was ordered to be taken every three hours ; and the saline draught was continued.

April 11. He had not been able to obtain much sleep. He said that he felt easier, and could breathe more freely. The skin was cool; the tongue was rather furred. A purgative draught was ordered.

April 12. He was much better to-day, and able to sit up. Respiration was free; there was no pain on making a full inspiration. There was some dulness near the wound and towards the left scapula; elsewhere, the respiration was free. The tongue was cleaner; the skin quite cool; the bowels freely open. The gums were beginning to be slightly affected. The pill was ordered to be taken at bed-time only.

April 13. He had a comfortable night. His gums were sore; and an alum gargle was ordered.

April 15. He was progressing favourably, and was ordered to have three ounces of wine, and half diet.

April 17. The patient was doing well. A tonic draught was prescribed.

April 19. Recovery was taking place rapidly. He complained of pain between the scapula; and near the inner border of the left, the bullet could be felt, but not superficially enough to warrant cutting down to it. A poultice was ordered to be applied to the part.

April 21. The bullet was still felt; but not more distinctly. In a day or two afterwards, no signs of the ball could be perceived; and the patient has been regaining his usual health up to the present date (May 20). He says that he feels some stiffness in the loins now and then. The aperture of the wound is closing, with slight discharge.

CASE II. Gun-shot wound of the Chest; Ball Lodged. W. Hutchinson, 3rd European Regiment, was admitted April 3, with a wound caused by a bullet, situated two and a half inches from the sternal end of the right clavicle, and one inch below 